\title{
El problema de la enseñanza de los marcadores discursivos y su visualización: sobre "bueno" en la conversación coloquial"
}

\author{
ADRIÀ PARDO LLIBRER \\ Grupo Val.Es.Co \\ adria.pardo@uv.es
}

Resumen: El presente trabajo reflexiona sobre la visualización de las funciones de los marcadores discursivos en el aula de E/LE, para lo que se toma como ejemplo la forma bueno. Esta visualización parte del modelo Val.Es.Co. para la segmentación del habla y articula las funciones de este marcador según su posición estructural y el ámbito sobre una determinada unidad discursiva. Esta metodología no solo resulta útil para la descripción lingüística, sino también para la enseñanza de los marcadores. La simplificación de esta metodología ofrece una visualización cuyas posibilidades, en último término, problematizamos.

Palabras clave: visualización, marcadores discursivos, modelo Val.Es.Co., bueno.

\section{On teaching and visualizing discourse markers: Spanish "bueno"}

Abstract: This paper deals with the visualization of the meanings of the Spanish discourse marker bueno ('well'). This visualization follows the Val.Es.Co. model for the discourse segmentation in units and positions. The application of this model is methodologically useful for the description of discourse markers, as well as for further teaching goals. Thus, two ways for the visualization of discourse markers are ultimately discussed.

Key words: visualization, discourse markers, Val.Es.Co. model, bueno.

\section{Introducción}

Este trabajo analiza las funciones del marcador discursivo bueno y las organiza según el lugar que esta forma ocupa en la conversación coloquial española. El objetivo es discutir algunas de las posibilidades que la visualización de los marcadores discursivos (en adelante, MD) ofrece en la enseñanza de E/LE. Para ello, se toma bueno como ejemplo, tanto por su polifucionalidad (Bauhr 1994, Martín Butragueño 2006), como por ser una forma propia de la oralidad (Pons 2003). En anteriores trabajos (Pardo Llibrer 2019, 2020), defendíamos la utilidad de la segmentación en unidades discursivas (de acuerdo con la propuesta de Grupo Val.Es.Co. 2014) para la enseñanza de estos elementos lingüísticos. Partiendo de la caracterización de bueno en el Diccionario de partículas discursivas del español (Briz, Pons y Portolés 2008; en adelante, DPDE), este trabajo se articula en tres puntos. En primer lugar $(\S 2)$, presentamos una serie de cuestiones teóricas preliminares: se expone nuestra concepción de los MD $(\S 2.1)$ y se

\footnotetext{
${ }^{1}$ Este artículo ha sido posible gracias al proyecto de investigación FFI2016-77842-P, Unidades discursivas para una descripción sistemática de los marcadores del discurso en español (UDEMADIS), financiado por el MINECO, la AEI y los fondos FEDER. 
introduce el modelo Val.Es.Co. como marco teórico más adecuado para el estudio de estos elementos ( $§ 2.2)$. En segundo lugar ( $(3)$, se exponen las cuestiones de orden metodológico: la aplicación del modelo Val.Es.Co. facilita una descripción de las funciones que desempeñan los $\mathrm{MD}(\S 3.1) \mathrm{y}$, en función del lugar que ocupan en la conversación coloquial $(\S 3.2)$, resulta una primera sistematización de sus principales usos según una posición discursiva u otra (\$ 3.3). Denominamos a esta sistematización parrilla $(\S 4)$, cuya aplicación didáctica $(\S 4.1)$ puede visualizarse tanto desde un punto de vista funcional como desde un punto de vista estructural (\$ 4.2). Unas conclusiones cierran el artículo $(\S 5)$.

\section{Cuestiones teóricas}

Dos son los aspectos teóricos clave para el tratamiento, en este trabajo, de los MD. Por una parte $(\S 2.1)$, una concepción estrictamente modular de los $\mathrm{MD}$, que entienda estos elementos como operativos únicamente en el plano pragmático de la lengua y por oposición a los elementos del plano semántico y/o gramatical. Por otra parte (§ 2.2), el estudio -de aspiración sistemática- de este plano pragmático donde operan los $\mathrm{MD}$, para lo que seguimos el modelo Val.Es.Co. de segmentación del habla. Sobre estas coordenadas se construye nuestra propuesta para la enseñanza de la marcación discursiva.

\subsection{Marcadores discursivos: naturaleza y función}

En términos generales, entendemos por MD aquellas formas suprasintácticas y extraproposicionales que desempeñan funciones pragmáticas. Es decir, se trata de elementos que no se rigen por su combinación con otros elementos del molde oracional -aunque pueden influir en ellos- ni afectan a su significado componencial, sino que guían las inferencias relativas a la interpretación del enunciado ( $c f$. Schiffrin 1987, Portoles 1999, Martín Zorraquino y Portolés 1999, Fraser 1999 entre otros). Por ejemplo ${ }^{2}$ :

(1) D: eso e- sí e- ee- comer en la naturaleza propia/ een (RISAS) la misma selva (RISAS)

B: en la misma mierda (RISAS)

C: bueno $\downarrow$ hemos ganao al fútbol ¿a qué nos vas a invitar?

A: os invito $\uparrow[$ a un bocao $\uparrow=]$

(Conversación H.38.A.1, intervenciones 675-676)

En la intervención del hablante $\mathrm{C}$, la utilización de bueno no depende de otros constituyentes sintácticos: este MD se sitúa en los así llamados márgenes oracionales y, en el caso de que fuera omitido, el contenido semántico del enunciado no variaría ${ }^{3}$.

\footnotetext{
2 Ejemplos extraídos del Corpus Valesco de Conversaciones Coloquiales (Briz y Grupo Val.Es.Co. 2002).

3 Esto se aprecia claramente si llevamos a cabo una descripción proposicional mínima de las oraciones implicadas en la intervención de $\mathrm{C}$ en el ej. (1). Si de la oración (i) se abstrae la proposición mínima (ii):

(1') i. bueno $\downarrow$ hemos ganao al fútbol (oración)

ii. bueno, hemos ganado al fútbol (proposición: forma lógica $P$ )
} 
Intuitivamente, podríamos decir que bueno introduce aquí un nuevo tema de conversación. Entendemos que esta función es pragmática porque es cancelable, o sea, porque permite inferir por dónde va $a$ ir el sentido del enunciado, sin alterar su significado en lo esencial (desde el punto de vista de la información transmitida). Ahora bien, esta no es la única función que bueno desempeña:

$$
\begin{aligned}
& \text { E: sí } \downarrow \text { pero desde pequeñito él ha estado en Montesinos/ interno } \\
& \text { L: ah } \\
& \text { E: luego cuando vino a Valencia } \uparrow / \text { bueno } \downarrow \text { cuando acabó el Cou/// [...] }
\end{aligned}
$$

(Conversación L.12.A.2, intervención 82)

En el ejemplo (2), bueno relaciona los enunciados entre los que se sitúa (cuando vino a Valencia cuando acabó el Cou), pero ninguno de los dos se explica desde la sintaxis. De hecho, este MD ayuda a inferir que el segundo enunciado es una manera alternativa de presentar la información del primero. Otro uso sería:

$$
\begin{aligned}
& \text { E: }[((¿ \text { cuántos capítulos tiene?))] } \\
& \text { G: que si la has leído o no la has leído } \\
& \text { E: no sé// bueno yo la Biblia } \uparrow \text { estoy hasta el gorro ya ¿eh?// yo he estao OCHO } \\
& \text { años con la Biblia encima }
\end{aligned}
$$

(Conversación L.12.A.2, intervención 329)

En el ejemplo (3), bueno ni relaciona ni introduce enunciados, sino que con él se pretende interferir en la interpretación de una información que, en este caso ('estoy hasta el gorro de la Biblia'), puede ser polémica. Se trata de una herramienta de la que dispone el hablante para hacer suyo lo dicho y, de este modo, evitar que se pueda entender como una afirmación general algo que es expresión particular. Por último, algunos usos de bueno se relacionan con contenidos enteramente implícitos, así:

$$
\text { A: ¿quieres un cigarrillo? }
$$

\section{B: bueno}

En el ejemplo (4), sin necesidad de decir sí, el hablante B se vale de bueno para orientar su intervención hacia una afirmación, por otra parte, no explicitada. En resumen, todos estos usos de bueno operan en un nivel diferente al gramatical, por lo que cabe acudir a una sintaxis de orden discursivo.

\subsection{El modelo Val.Es.Co y el estudio de los marcadores discursivos}

Tal y como los definimos, los MD son elementos operativos en el plano discursivo. En este sentido, las categorías que se precisan para su análisis han de ser de base pragmática. En este trabajo, nos apoyamos en el modelo Val.Es.Co. para la segmentación en unidades discursivas y posiciones (Grupo Val.Es.Co. 2014) ${ }^{4}$. Este

\footnotetext{
iii. 'GANAR'(nosotros, fútbol) (forma proposicional)

se obtiene una formulación proposicional (iii) en la que bueno no tiene cabida, pues no forma parte de los constituyentes de significado susceptibles de recibir una asignación de valores de verdad.

${ }^{4}$ Todos los conceptos del modelo, en este epígrafe, remiten a Grupo Val.Es.Co. (2014).
} 
modelo persigue una segmentación del habla, explicativa -entre otros fenómenos ${ }^{5}-$ del funcionamiento de los marcadores discursivos.

El modelo Val.Es.Co. se articula en torno a dos niveles y tres dimensiones:

\begin{tabular}{|l|l|l|l|}
\hline \multicolumn{1}{|c|}{ NIVELES } & \multicolumn{3}{|c|}{ DIMENSIONES } \\
\hline Dialogal & $\begin{array}{l}\text { ESTRUCTURAL } \\
\text { Discurso } \\
\text { Diálogo } \\
\text { Intercambio }\end{array}$ & INFORMATIVA \\
\hline Monologal & $\begin{array}{l}\text { Intervención } \\
\text { Acto }\end{array}$ & Turno & Subacto \\
\hline
\end{tabular}

Figura 1. Niveles y dimensiones del modelo Val.Es. Co. (Grupo Val.Es.Co. 2014)

Tal y como consta en la Fig. 1, son ocho (en cursiva) las unidades de análisis del modelo. Estas unidades se articulan en torno a dos niveles (dialogal y monologal) y tres dimensiones (estructural, social e informativa). El nivel dialogal hace referencia a la implicación de dos o más hablantes y el monologal, a lo dicho solamente por uno. En cuanto a las dimensiones: la dimensión estructural tiene que ver con las posibles divisiones que un segmento lingüístico puede recibir, la dimensión social engloba las unidades que tienen que ver con el papel de los interlocutores en una conversación ${ }^{6}$ y la dimensión informativa, con la naturaleza proposicional o no de segmentos inferiores.

Por motivos expositivos, nos centramos para el estudio de los MD en las unidades de las dimensiones estructural e informativa, ya que permiten describir relaciones entre formas y segmentos lingüísticos, más allá del uso que de estos hagan los hablantes. Sucintamente, estas unidades pueden definirse como sigue:

a. Discurso (DSC): un discurso en sí, con un tema y unos papeles sociolingüísticos dados para los hablantes, entendido como un fluir conversacional que cambia cuando cambian su tema y sus papeles sociolingüísticos.

b. Diálogo (DI): una sucesión de intervenciones de dos o más hablantes en torno a uno de los temas desplegados en el discurso.

c. Intercambio (INT): par mínimo de interacción entre dos hablantes, dos contribuciones a la conversación relacionadas entre sí.

d. Intervención (I): contribución de un hablante a la conversación, lo que este dice.

e. Acto (A): unidad mínima de acción comunicativa, que tiene la propiedad de aislarse como una intervención propia ${ }^{7}$.

f. Subacto (S): unidad mínima informativa, introduce algún tipo de contenido, que puede ser o bien semántico (proposicional) o bien pragmático (inferencial) ${ }^{8}$.

\footnotetext{
${ }^{5}$ Atenuación (Albelda 2010, Briz 2007), gramaticalización (Estellés 2011), elementos subestructurales (Pascual Aliaga 2018), fórmulas conversacionales (Salameh 2020), etc.

${ }^{6}$ Esta dimensión incluye las unidades determinadas por la condición de los hablantes en calidad de entes sociales. Esto es, la unidad turno, entendida como una intervención reconocida por el resto de interlocutores en la conversación, y la unidad intercambio de turnos, el paso de un hablante a otro en la posesión del turno de palabra.

${ }^{7}$ De manera más detallada, el acto se define según tres criterios: un criterio pragmático (todo acto posee una fuerza ilocutiva distintiva), un criterio semántico (todo acto constituye una forma lógica mínima) y un criterio prosódico (todo acto se integra o conforma un grupo entonativo).
} 
Las tres últimas unidades son especialmente relevantes para nuestros fines. Por ejemplo:

(1') C: \#bueno $\downarrow$ hemos ganao al fútbol\# \# ¿a qué nos vas a invitar?\#

Este ejemplo constituye una intervención (contribución de $\mathrm{C}$ a la conversación), al tiempo que se puede segmentar en dos actos (segmentados mediante almohadillas: \#...\#). Se entiende, a grandes rasgos, que un segmento como hemos ganao al fútbol presenta una fuerza ilocutiva diferente a la de la pregunta ¿a qué nos vas a invitar? que le sigue. Además, cada segmento tiene entidad suficiente como para, hipotéticamente $\left(1 '\right.$ '), constituirse en una intervención congruente $\left(C^{\prime}\right)$ :

(1'’) C: \#bueno $\downarrow$ hemos ganao al fútbol\#

$$
\begin{aligned}
& \text { X': me toca invitar a una ronda } \\
& \text { C': \# ¿a qué nos vas a invitar?\# }
\end{aligned}
$$

Es frecuente que un mismo acto se pueda dividir en dos o más subactos (segmentados entre claves: $\{\ldots\}$ ). Se distinguen dos tipos: los subactos sustantivos (SS), con contenido proposicional; y los subactos adyacentes (SA), extraproposicionales y supeditados al contenido semántico -y, así, nuclear- de un subacto sustantivo. Por ejemplo:

(3') E: \#no sé//\# \#\{sa bueno sa\} \{ss yo la Biblia $\uparrow$ ss\} \{ss estoy hasta el gorro ya ss\} $\{$ sa $¿$ eh?// sa $\} \#$ \#yo he estao OCHO años con la Biblia encima\#

Así, bueno depende de la información aportada por los SS ('estoy hasta el gorro de la Biblia'), al tiempo que la enriquece (esto es, su uso no tiene sentido en el enunciado por sí solo). La unidad subacto, entonces, presenta diferentes caracterizaciones funcionales según su relación con otros subactos:

- Los SS pueden subdividirse en subactos sustantivos directores (SSD), con contenido semántico nuclear; subactos sustantivos subordinados (SSS), dependientes de los SSD; y subactos sustantivos topicalizados (SSSTop), dislocaciones pragmáticas prosódicamente separadas y no explicables sintácticamente.

- Por su parte, los SA pueden ser subactos adyacentes textuales (SAT), cuando contribuyen a organizar la información; subactos adyacentes modalizadores (SAM), si invitan a una interpretación subjetiva de lo dicho; y subactos adyacentes interpersonales (SAI), si implican alguna relación entre hablante y oyente.

De acuerdo con esto, la segmentación del ejemplo (3') se puede describir con mayor detalle, de cara a estudiar la función que desempeña cada segmento lingüístico en el conjunto de la intervención. Luego:

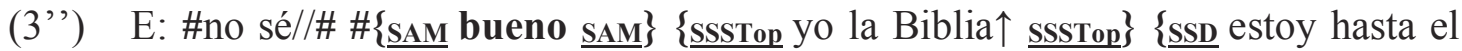
gorro ya $\underline{\mathbf{S S D}}\}\{\underline{\mathbf{S A I}} i$ eh?// $\underline{\mathbf{S A I}}$ \#\# \#yo he estao OCHO años con la Biblia encima\#

\footnotetext{
${ }^{8}$ Se habla también -al modo de la teoría de la relevancia- de subactos que aportan un contenido conceptual (léxico) frente a un contenido procedimental (pragmático, en sentido amplio). Sin embargo, preferimos evitar esta nomenclatura, ya que es conceptualmente problemática.
} 
Como vemos, el segundo acto de esta intervención se caracterizaría por tener un SSD, en tanto que unidad nuclear, y un SSSTop supeditado 9 . Aparte de esto, el ejemplo (3"') da cuenta de la importancia de los subactos adyacentes (SA) en el estudio de los MD. En calidad de elementos discursivos que guían las inferencias de un enunciado, sus funciones se pueden explicar según estas sean textuales, modales o interactivas: aquí bueno modaliza un contenido polémico, ya que tiene ámbito sobre todo el acto; mientras que ¿eh?, con el mismo ámbito, permite interpelar la atención del otro hablante. De acuerdo con este ejemplo, ambos MD coinciden en el ámbito, pero difieren en su posición. La consideración de las posiciones, en relación con las diferentes unidades sobre las que un MD puede tener ámbito, así como el paralelismo entre funciones pragmáticas y tipo de SAT/SAM/SAI, redundan en las cuestiones metodológicas para su análisis.

\section{Cuestiones metodológicas}

El modelo Val.Es.Co. distingue tres funciones macro $^{10}$ para los SA, las cuales podemos reproducir en la caracterización de los MD. A saber: macrofunciones textual (SAT), modalizadora (SAM) e interpersonal (SAI). Como subactos adyacentes, tienen ámbito sobre una unidad sustantiva o superior (SSD, actos, intervenciones...), al tiempo que influyen en estas, induciendo una inferencia que connota un sentido $\mathrm{u}$ otro al enunciado en conjunto. Estas unidades son categorías funcionales, no formales, por lo que su descripción no puede ser en modo alguno automática, sino que exige el análisis de cada ocurrencia en particular. En esta descripción, se tiene en cuenta un último factor: la posición de un MD como indicio distintivo (Briz y Pons 2010). Así, un MD podría ocupar hasta cinco posiciones objetivas:

a. Inicial - [I]: el MD ocupa una posición inicial cuando es el primer elemento de la unidad sobre la que tiene ámbito.

b. Inicial relativa - [IREL]: el MD ocupa una posición inicial relativa cuando se encuentra al inicio de la unidad, pero puede estar antecedido por otros conectores.

c. Medial - $[\mathrm{M}]$ : el MD ocupa una posición medial cuando aparece dentro de la unidad sustantiva, pero sin formar parte de esta.

d. Final - [F]: el MD ocupa una posición final cuando es el último elemento de la unidad sobre la que tiene ámbito.

e. Independiente - [IND]: el MD constituye el único elemento explícito de la intervención.

Esta nómina de posiciones tiene, para los fines del presente trabajo, un carácter únicamente descriptivo. Sin embargo, en relación con una u otra unidad discursiva, las posiciones son indicios significativos, por lo que -en principio- no se descarta su valor explicativo.

\footnotetext{
${ }^{9}$ Sin poder profundizar en esta cuestión, se trataría de un SSSTop y no de una focalización gramatical, puesto que la rección gramatical se suspende y la entonación queda marcada por un tonema ascendente.

${ }^{10}$ En cierto modo, a la manera de Halliday y Hasan (1976), quienes oponen a la función ideacional (semántica), las funciones textual (SAT) e interpersonal (en Val.Es.Co., SAI y también SAM).
} 


\subsection{Abstracción de funciones}

Asumiendo estas posiciones para los subactos adyacentes, se pueden perfilar aquellos huecos funcionales más recurrentes en el habla para un MD (según el ámbito que dicho MD tenga sobre una unidad). Esto nos permite identificar las funciones básicas del MD bueno, partiendo de una interpretación inicial que se vea perfilada por una objetivación según unidad y posición:

(5) (Hablando de un reloj encontrado en la calle)

B: sí yooo hoombre yo pienso en principio si sería de oro

A: \# DE ORO $\downarrow$ qué va $\downarrow$ bueno $\downarrow$ la correa sí que era $\uparrow$ \# [...]

El $D P D E^{11}$, en un ejemplo como (5), considera que el MD bueno "rectifica la información inmediatamente anterior, que indicaba que ninguna parte del reloj era de oro". Este proceso es lo que se conoce como reformulación (Roulet 1987, Gülich y Kotschi 1995, Rossari 1994, Pons 2013). Se trata de una función textual (SAT) que relaciona dos segmentos, un primer segmento reformulado (lo rectificado) y un segmento reformulador (la rectificación, introducida por bueno). En una segmentación en unidades, el primer segmento es un SSS ('el reloj no era de oro'), dado que depende enteramente del segundo segmento reformulador ('una parte del reloj era de oro'), que sería el SSD. Luego:

(5') (Hablando de un reloj encontrado en la calle)

B: sí yooo hoombre yo pienso en principio si sería de oro

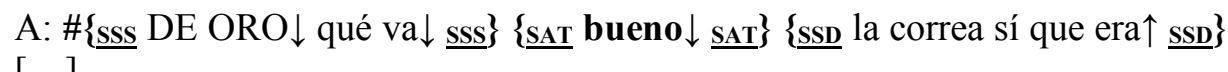
$[\ldots]$

Así pues, la función reformuladora de bueno puede caracterizarse como una función textual, en posición inicial de SSD (en notación: [I, SSD]). Claro está, no todas las funciones presentan la misma posición:

(6) — ¡Hombre! ¿No se puede silbar? —preguntó Martín.

-No, señor.

\# Bueno. No silbaré. \#

Esta función se entiende en el DPDE como una "matización" sobre lo dicho, esto es, un uso que tiene ámbito sobre el conjunto del acto: la naturaleza ilocutiva de esta unidad permite que bueno modalice el enunciado (se trata de un SAM), a modo de estrategia atenuadora. Se presta, así, a la siguiente segmentación:

(6’) — ¿Hombre! ¿No se puede silbar? —preguntó Martín.

-No, señor.

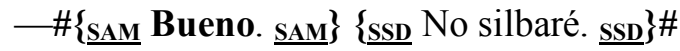

Aquí bueno constituye un SAM con ámbito sobre todo el acto que encabeza (en notación: [I, A]). Ahora bien, así como un MD no presenta una única función, tampoco todas sus funciones se dan en una única posición, es decir, la distinción última depende del

\footnotetext{
${ }^{11}$ Los ejemplos problematizados, así como las citas no especificadas en este epígrafe, se corresponden con la entrada de bueno en el DPDE (Pons 2008; en Briz, Pons y Portolés 2008).
} 
alcance de la función pragmática introducida por bueno. Asumiendo esta función atenuante o de "matización", la posición, con respecto a (6), varía en un ejemplo como (7):

(7) A: \# no/ yo me pude reír con un programa de bueno/ el de Hola Rafaela/ que l'han puesto a(ho)ra nue- otra veh \#

El MD, en este caso, vendría a repercutir sobre el conjunto del acto pero, en posición medial (en notación: [M, A]), su ámbito se focaliza sobre un segmento menor (el programa de Hola Rafaela) parte del SSD:

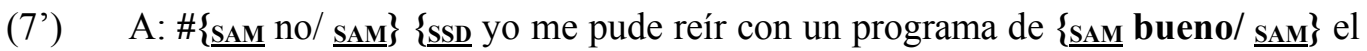
de Hola Rafaela/ que l'han puesto a(ho)ra nue- otra veh $\underline{\mathbf{S s D}}$ \}\#

Otras funciones, por el contrario, extienden su ámbito sobre unidades superiores:

(8) S: ¿cómo que no hay baña-? ¡ah bueno! que no es la época

L: pero en los grandes almacenes [síi=]

S: [claro]

$\mathrm{L}:=$ que suelen haber

B: \# bueno hay/ pero de esos de natación \#

El DPDE entiende que un uso como (8) "[i]ndica acuerdo, total o parcial, con algo dicho anteriormente"; se trata de una función interpersonal (SAI), con ámbito sobre toda la intervención del hablante $\mathrm{B}$ y necesariamente relacionada con la intervención previamente $^{12}$ formulada por el hablante L. Se trata de una posición inicial de intervención (en notación: [IND, I]) y arroja la siguiente segmentación:

(8) S: ¿cómo que no hay baña-? ¡ah bueno! que no es la época

L: pero en los grandes almacenes [síi=]

S: [claro]

$\mathrm{L}:=$ que suelen haber

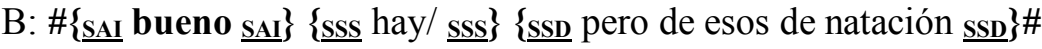

Por otra parte, con esta función de acuerdo, el MD bueno puede ocupar también una posición independiente (en notación: [IND, I]):

(9) (dos vecinas están hablando sobre la conveniencia de instalar o no un ascensor en el edificio)

A: los [vecinos] de- ahora le han dao permiso/ ahora lo han puesto

M: ¡Ay! ¿vees?§

A:

$\S$ bueno§

\footnotetext{
${ }^{12}$ Sin profundizar en esta cuestión, son el tipo de intervenciones que el modelo Val.Es.Co. denomina iniciativoreactivas (responden a una contribución anterior, al tiempo que garantizan la continuidad del diálogo), por oposición a las intervenciones solamente iniciativas (que inauguran un diálogo) y a las intervenciones solamente reactivas (respuestas fáticas $-s i ́$, aha- o de control del contacto $-y a$, claro- sin posibilidad de constituirse en turno).
} 
Comoquiera que denominemos los diversos usos observados, bueno se revela como un MD claramente polifuncional, en la medida en que presenta diferentes funciones que, a su vez, son de diferente naturaleza pragmática (textuales, modalizadoras e interpersonales). La descripción de estas funciones, en cualquier caso, es susceptible de sistematización.

\subsection{Parrilla de unidades por posición: de la descripción al metalenguaje}

Como se ha expuesto en trabajo anteriores (Pardo Llibrer 2020), es posible llevar a cabo una sistematización de las principales funciones que un MD dado puede desarrollar. Para ello, procedemos al análisis de bueno mediante el cruce de tres variables:

1. En primer lugar, se identifican las funciones descritas en el $D P D E$ y se traducen a un metalenguaje específico (alejándonos de las generalizaciones más o menos imprecisas que se ofrecen a los usuarios no especialistas). Una vez identificadas estas funciones (reformulación, atenuación, acuerdo), las abstraemos -como arriba- en uno u otro tipo de subacto adyacente.

2. Seguidamente, aplicando el modelo Val.Es.Co., se segmentan los ejemplos en los que el MD aparece. De esta manera, a cada función se le puede atribuir una unidad discursiva (SSD, acto, intervención...) sobre la que tiene ámbito. Ni que decir tiene que no es imposible que dos funciones difieran en su naturaleza pragmática (p. ej., que un MD pueda desempeñar la función de SAT y de SAM), pero coincidan en la unidad sobre la que tienen ámbito; en estos casos, el rasgo distintivo lo marca el tipo de subacto adyacente.

3. El último paso consiste en describir la posición que el MD analizado ocupa con respecto a las unidades discursivas resultantes de la segmentación. Para ello, es necesario identificar el segmento sobre el que MD influye (lo que se puede formular mediante las notaciones arriba ya introducidas: [I, SSD $],[\mathrm{M}, \mathrm{A}] \ldots)$.

Teniendo esto en cuenta, se pueden disponer en un eje vertical las posiciones contempladas en el modelo y, en otro eje horizontal, las unidades sobre las que -en este caso- el MD bueno puede tener ámbito. A partir de este cruce, se dibuja una tabla con las diferentes celdas en las que insertar las funciones descritas para bueno. El resultado es el que sigue:

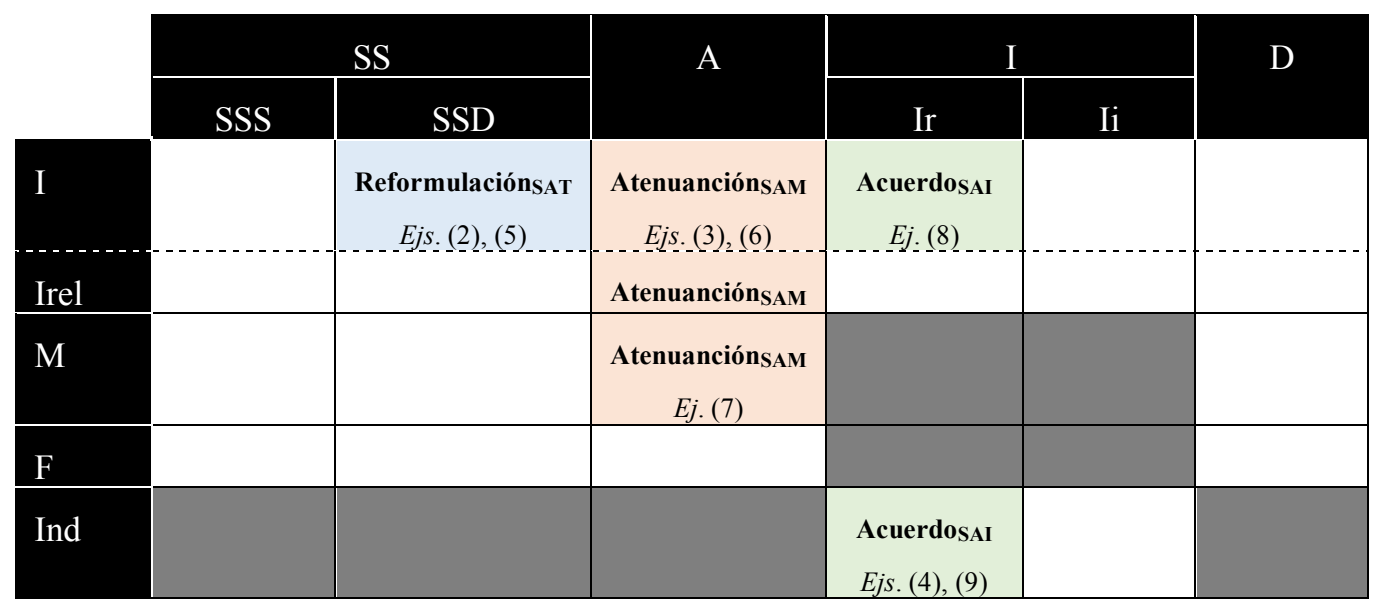

Figura 2. Parrilla de funciones de bueno 
Se obtiene, así, lo que se denomina parrilla de funciones según unidades por posición o, sencillamente, parrilla de funciones. Los espacios sombreados suponen las posiciones estructuralmente imposibles o poco probables dentro del modelo y el resto de los huecos, los lugares donde situar las funciones descritas. Así, las funciones de los ejemplos tratados se corresponden, al menos en la descripción de este MD, con posiciones especializadas según el tipo de SAT/SAM/SAI. En este sentido, bueno presenta una peculiaridad descriptiva hasta cierto punto poco común en el proceso de elaboración de parrillas: cada macrofunción tiene lugar en una unidad diferente, a lo que hay que añadir una escasa variación en las posiciones (tanto es así que una función como la reformulación está encasillada en una única coordenada unidad posición).

\subsection{Adaptación de las parrillas: de lo metalingüístico a lo aplicado}

No pasa inadvertida la complejidad de esta parrilla de funciones según unidades por posición. La parrilla de la Fig. 2 constituye una descripción de orden teórico, destinada a la investigación de la marcación discursiva. Ahora bien, el metalenguaje de esta parrilla se puede simplificar paulatinamente, hasta alcanzar ciertas aplicaciones. En otras palabras, la Fig. 2 es útil para el lingüista, pero un tanto opaca para un potencial docente de E/LE poco familiarizado con el modelo Val.Es.Co. (algo, por otra parte, más que probable). Por ello, conviene reducir la prolijidad tanto de unidades como de posiciones. Los cambios que se introducen son los siguientes:

- La unidad intervención se puede redefinir con el término más intuitivo de turno ${ }^{13}$ y el acto, con el término genérico -y más amplio- de enunciado.

- El término oración se refiere a las unidades con mayor integración sintáctica (es decir, SSS y SSD) y el término cláusula, a constituyentes menores sobre los que un MD puede poner el foco (esto es, partes de subacto).

- Por útlimo, la posición inicial y la inicial relativa se pueden reducir a una. Luego:

\begin{tabular}{|c|c|c|c|c|}
\hline & Cláusula & Oración & Enunciado & Turno \\
\hline INICIO & & Reformulación & Atenuación & Acuerdo \\
\hline MITAD & & & Atenuación & \\
\hline FINAL & & & & \\
\hline INDEP. & & & & Acuerdo \\
\hline
\end{tabular}

Figura 3. Parrilla para el profesor

La visualización metalingüística de la Fig. 2 se reduce, así, a las categorías de la Fig. 3, de suerte que puede funcionar a modo de guía; bien para que el docente desarrolle sus propias aplicaciones, bien para que le sirva de material con que introducir a los alumnos en una versión de la parrilla más simplificada si cabe, como se expone a continuación.

${ }^{13} \mathrm{Si}$ bien no en el sentido de las unidades de la dimensión social del modelo Val.Es.Co. (véase nota 6). 


\section{Cuestiones didácticas}

El paso de la parrilla descriptiva (Fig. 2) a la parrilla para el profesor (Fig. 3) plantea el problema de una visualización de la relación de funciones para bueno. En palabras de Espinosa Guerri (2016: 14), en relación con los procedimientos de visualización: “el investigador ya no se enfrenta directamente al bloque de información [...] sino que dispone de un mecanismo que le permite estructurar toda esa información, establecer relaciones concretas y jerarquizar los elementos". Para llegar a este mecanismo que pueda ser útil para los alumnos, adaptamos un grado más nuestra parrilla.

\subsection{Correspondencia entre parrillas y ejemplificación}

El objetivo al que aspiramos es una parrilla mínima que, en primer lugar (§ 4.2.1), pueda servir de herramienta de consulta metalingüística para el alumno $\mathrm{y}$, en segundo lugar (§ 4.2.2), se corresponda con ejemplos concisos y precisos.

\subsection{1. ¿Una parrilla para los alumnos?}

El paso de la parrilla de la Fig. 2 a la de la Fig. 3 consiste básicamente en reducir categorías y renombrarlas de modo más transparente. En el paso de una parrilla para el profesor a otra para el alumno, cabe también simplificar el cómo presentamos las funciones. La parrilla se transforma del siguiente modo:

- La unidad enunciado se subsume en la de oración, más familiar para el estudiante; mientras que la unidad cláusula se renombra como parte de oración y turno, como respuesta (fácilmente asociable por su carácter dialogal).

- Las posiciones se mantienen en lo esencial, cambiando la denominación de independiente por la de solo.

- En cuanto a las funciones, estas se traducen a términos finalistas. Esto es, en lugar de hablar de funciones, estas se representan como el para qué más habitual de los actos de habla en que aparecen. Así, para el alumno, la función de reformulación se equipara a para cambiar; la de atenuación, a para suavizar; y la de acuerdo, a para aceptar. Todo ello se traslada a la parrilla modificada:

\begin{tabular}{|c|c|c|c|}
\cline { 2 - 4 } & Partedeoración & Oración & Respuesta \\
\hline Inicio & Para cambiar & Para suavizar & Para aceptar \\
\hline Mitad & & Para suavizar & \\
\hline Final & & & \\
\hline Solo & & & Para aceptar \\
\hline
\end{tabular}

Figura 4. Parrilla para el alumno

Esta traducción finalista de las funciones entronca con una disposición orientativa de ejemplos que pueda acompañar a cada parrilla. 


\subsubsection{Ejemplos ad hoc y claridad didáctica}

Para la ejemplificación de las funciones de la parrilla del alumno, cabe buscar ejemplos cuyo contenido sea lo más transparente posible para el estudiante, pero fácilmente reconocibles como elementos en la interacción. Podemos esbozar una primera ejemplificación:

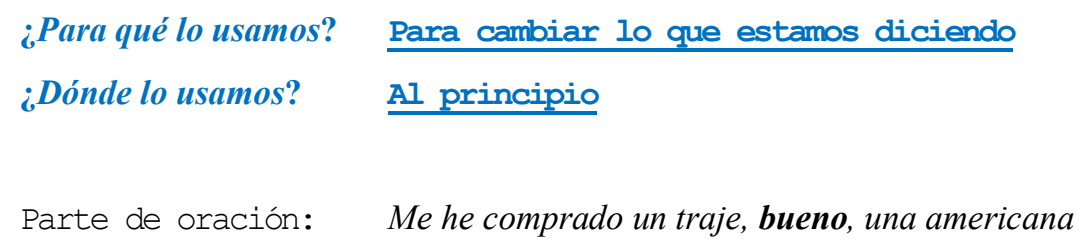

¿Para qué lo usamos? Para suavizar lo que decimos

¿Dónde lo usamos? Al principio $y$ en el medio

Oración: $\quad$ A: $\quad-O y e$ el otro día no viniste a la comida, ¿verdad?

B: -Bueno, últimamente tengo muchos exámenes

-Últimamente... bueno, tengo muchos exámenes

¿Para qué lo usamos?

¿Dónde lo usamos?

Para aceptar alguna cosa

Al principio y solo

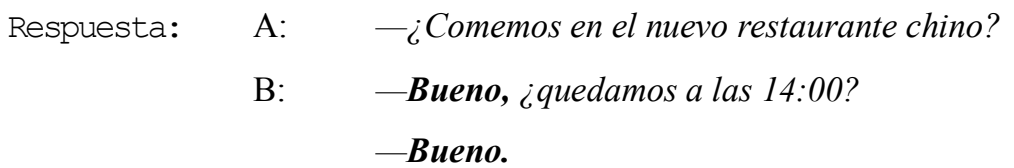

Para esta ejemplificación, se establece una correspondencia cromática entre las tres funciones (SAT en azul, SAM en rojo y SAI en verde) y los ejemplos ad hoc. Estos ejemplos se representan dentro de contextos de uso, por así decirlo, prototípicos. El ejemplo para la reformulación (para cambiar), se reproduce en una enunciación monologal con ámbito sobre segmentos -un traje y una americana- sintácticamente integrados; por su parte, los ejemplos de atenuación (para suavizar) y acuerdo (para aceptar), se han de mostrarse necesariamente en enunciaciones dialogales.

Hasta aquí, el resultado de esta parrilla, acompañada de un modelo de ejemplificación práctica para los alumnos, se ajusta al marcador bueno. Teóricamente, cabría concluir que esta visualización es replicable para otras formas: las funciones textuales extienden su ámbito sobre la parte de oración, las modales sobre la oración y las interpersonales sobre la respuesta. Por el contrario, un MD ni presenta una única función, ni todas sus funciones presentan siempre una posición privativa según sean SAT/SAM/SAI, como ocurre con bueno. Puede ocurrir, pues, que algunos MD no permitan una descripción estructural donde función y posición se puedan visualizar biunívocamente. Este hecho reverbera en una cuestión de orden didáctico, como es el tipo de visualización de las parrillas, y que a continuación problematizamos. 


\subsection{Sobre la visualización funcional y la visualización estructural}

La descripción simplificada para el aula de E/LE se puede ajustar a dos visualizaciones posibles: una visualización que denominamos estructural y otra que denominamos funcional. En rigor, toda visualización es en última instancia funcional, puesto que se centra en los usos más recurrentes de un MD. Sin embargo, cuando la descripción revela unas funciones especializadas posicionalmente, la visualización -como ocurre con bueno- es además estructural. Por consiguiente, se deducen dos posibles soluciones a esta situación: una solución heterogénea ( $\S 4.2 .1)$, que describa las funciones sea cual sea su correspondencia posicional; o una solución homogeneizadora ( $\S 4.2 .2$ ), que adapte la descripción a la parrilla.

\subsubsection{Una solución heterogénea}

La solución heterogénea se sigue de la descripción original de las funciones del MD: según el MD que estemos tratando, será necesaria una visualización que distinga las diferentes funciones que se den en una misma posición. Así, en un primer trabajo sobre pues (Pardo Llibrer 2019), el análisis arrojaba una parrilla del siguiente tipo:

\begin{tabular}{|c|c|c|c|}
\hline & Parte de oración & Oración & Respuesta \\
\hline Inicio & Para seguir & \multirow{2}{*}{ Para suavizar } & Para empezar \\
\hline Mitad & & & \\
\hline \multicolumn{4}{|l|}{ Final } \\
\hline Solo & & & \\
\hline
\end{tabular}

Figura 5. Parrilla de pues

En este caso, las funciones textuales (para seguir: continuación y formulación) y modalizadoras (para suavizar: atenuación) de pues coinciden en su posición y su ámbito sobre una misma unidad discursiva (Fig. 5, espacio sombreado). La solución heterogénea, entonces, invita a describir estos casos mediante una parrilla, por así decir, menos limpia que la de un MD como bueno, en cuya visualización las funciones no se solapan.

\subsubsection{Una solución homogénea}

Cabe, no obstante, una alternativa a esta heterogeneidad. Si la visualización funcional supone una solución heterogénea, una visualización estructural para cualquier MD persigue un formato único para todas las parrillas. Si bien no podemos dar una respuesta concluyente a este problema, sí se pueden esbozar algunas directrices para asumir esta labor:

- En primer lugar, hay que asumir que dos funciones de naturaleza diferente (p. ej., en pues, una textual y otra modalizadora) difícilmente se pueden colapsar en una única categoría descriptiva; sin embargo, para incluirlas indistintamente en una misma posición, es necesario aglutinarlas bajo una única etiqueta didáctica. 
- Así, en segundo lugar, es necesario igualar terminológicamente las dos funciones coincidentes, a fin de ceñirnos a una sola celda en la parrilla. Para esto, optamos por la traducción de las funciones a términos finalistas: nuestra solución a la coincidencia-Fig. 5- entre la función formulativa de pues (hablar para no perder el turno) y la función atenuadora (matizar lo que se está diciendo) es la de encontrar una etiqueta que pueda recoger ambos usos.

Tal etiqueta será descriptivamente poco rigurosa, pero su valor se ha de medir en función de lo intuitiva que le resulte al alumno. En tanto que la función formulativa mantiene el turno hasta concluir qué se va a decir y la función atenuadora evalúa lo que se va a decir como polémico, nuestra propuesta para estos usos de pues es la etiqueta finalista para pensar:

\begin{tabular}{|c|c|c|c|}
\cline { 2 - 4 } \multicolumn{1}{c|}{} & Partedeoración & Oración & Respuesta \\
\hline Inicio & Para seguir & Para pensar & Para empezar \\
\hline Mitad & & Para pensar & \\
\hline Final & & & \\
\hline Solo & & & \\
\hline
\end{tabular}

Figura 6. Reajuste de pues

En efecto, esta visualización estructural es incoherente con una correspondencia ideal con SAT/SAM/SAI, pero permite mantener la pauta seguida para bueno, al menos en lo que toca a la percepción una función una posición que queremos conservar. Por último, después de este proceso de abstracción, conviene dar con la nomenclatura que mejor oponga la finalidad de una posición a otra: la propuesta para pensar tal vez no sea la más transparente, pero ayuda a distinguir estos usos de los usos para seguir (función continuadora) y de los usos para empezar (función de inicio de tema).

\section{Conclusiones}

En definitiva, la descripción de un MD según unidades discursivas y posiciones ofrece una parrilla que puede simplificarse en función de las necesidades del usuario. A la hora de idear una versión para el alumno, existe la posibilidad de primar en su visualización o bien las funciones, o bien la posición. La visualización funcional es la más coherente con la parrilla metalingüística original, pero implica un mayor número de variables que el estudiante forzosamente habría de considerar. La visualización estructural, por su parte, resulta en unos MD y en otros no, pero pensamos que estos últimos casos -pese a la solución un tanto sui géneris aquí sugerida- no son insalvables. El reto, al que se habrá de hacer frente en investigaciones posteriores, es el de encontrar una metodología cerrada con que poder articular cualquier MD en una visualización estructural.

\section{Bibliografía}

Albelda MARCO, Marta. 2010. «¿Cómo se reconoce la atenuación?». (Des)cortesía en español. Roma: Programa EDICE, 41-70. 
BAUHR, Erhard. 1994. «Funciones discursivas de bueno en español moderno», Lingüística Española Actual, 16, 79-124.

BRIZ, Antonio y GRUPO VAL.ES.CO. 2002. Corpus de conversaciones coloquiales (Anejo I de la revista Oralia), Madrid, Arco-Libros.

BRIZ, Antonio, Pons, Salvador y Portolés, José. 2008. Diccionario de partículas discursivas del español. En línea: http://dpde.es

BRIZ, Antonio. 2007. «Para un análisis semántico, pragmático y sociopragmático de la atenuación en España y América». Lingüistica Española Actual 29 (1), 5-44

BRIZ, Antonio y PONS BORDERÍA, Salvador. 2010. «Unidades, marcadores discursivos y posición». En Loureda, Óscar \& Acín, Esperanza (dirs.), Los estudios sobre marcadores del discurso, hoy. Madrid, Arco/Libros, pp. 523-557.

ESPINOSA GUERRI, Guadalupe 2016. «Dientes de sierra: una herramienta para el estudio de la estructura interactiva del discurso dialógico», Normas, 6, 13-27.

ESTELlÉS, Maria 2011. Gramaticalización y paradigmas: un estudio a partir de los denominados marcadores de digresión en español. Fráncfort Meno: Peter Lang.

FRASER, Bruce (1999). «What are discourse markers?», Journal of Pragmatics, 31, 931-952.

GRUPO VAL.ES.CO. 2014. «Las unidades del discurso oral. La propuesta Val.Es.Co. de segmentación de la conversación (coloquial)». Estudios de Lingüistica del Español, 35, 13-73.

GÜLICH, Elisabeth y Thomas KOTSCHI. 1983. «Les Marqueurs De La Réformulation Paraphrastique», Cahiers de linguistique francaise, 5, 305-51.

HALliDAY, Michael Alexander Kirkwood y Ruqaiya HASAN. 1976. Cohesion in English. Londres: Longman.

Martín ButragueÑo, Pedro. 2006. «Prosodia del marcador bueno», Anuario de Letras, 44, $17-76$.

MARTÍN ZORRAQUINO, M. ${ }^{\text {a }}$ Antonia y PORTOLÉS, José. 1999. «Los marcadores del discurso». En I. Bosque \& V. Demonte (dirs.), Gramática descriptiva de la lengua española. Madrid: Espasa Calpe, vol.3, 4051-4213.

PARDO LLIBRER, Adrià. 2020. «La polifuncionalidad de los marcadores discursivos en E/LE según unidad y posición», Foro de Profesores de E/LE, 16, 275-286.

PARDO LLIBRER, Adrià. 2019. «La enseñanza de marcadores del discurso en el aula de E/LE desde la segmentación en unidades de la sintaxis del habla: el caso de 'pues'», Foro de Profesores de E/LE, 15, 275-286.

PASCUAL AliAgA, Elena. 2018. «Análisis prosódico de las estructuras truncadas en la conversación coloquial española: funciones de formulación y atenuación», ELUA: Estudios de atenuación en el discurso (Anexo IV), 57-84.

PONS BORDERÍA, Salvador. 2013. «Un solo tipo de reformulación», Cuadernos AISPI, 2, 151170.

PONS BorderíA, Salvador. 2008. «Bueno». En A. Briz, S. Pons y J. Portolés (dirs.), Diccionario de partículas discursivas del español. En línea: http://www.dpde.es/\#/entry/bueno1

PONS BORDERÍA, Salvador. 2003. «From agreement to stressing and hedging: spanish bueno and claro». En Gudrun Held (dir.), Partikeln und Höfflichkeit. Berna: Peter Lang, 219-236.

PORTOLÉs, José. 1998. Marcadores del discurso. Barcelona: Ariel. 
ROSSARI, Corinne. 1994. Les opérations de reformulation: analyse du processus et des marques dans une perspective contrastive française-italien. Berna: Peter Lang.

ROULET, Eddy. 1987. "Completude interactive et connecteurs reformulatifs», Cahiers de linguistique française, 8, 111-140.

SALAMEH JiMÉNEZ, Shima. 2020. «Pues eso como construcción interactiva desde el modelo Val.Es.Co.», Zeitschrift für Katalanistik, 33, 99-130.

SCHIFFRIN, Deborah. 1987. Discourse markers. Cambridge: Cambridge University Press. 